\title{
Lower IQ is Associated with Decreased Clinical Response to Atomoxetine in Children and Adolescents with Attention-Deficit Hyperactivity Disorder
}

Luigi Mazzone, ${ }^{1}$ Laura Reale, ${ }^{1}$ Valeria Mannino, ${ }^{1}$ Mariadonatella Cocuzza ${ }^{1}$ and Benedetto Vitiello ${ }^{2}$

1 Division of Child Neurology and Psychiatry, Department of Pediatrics, University of Catania, Catania, Italy

2 Child and Adolescent Treatment and Preventive Intervention Research Branch, National Institute of Mental Health, Bethesda, Maryland, USA

Objectives: Atomoxetine is commonly used to treat attention-deficit hyperactivity disorder (ADHD) in children with a broad range of cognitive abilities. We examined the association between level of cognitive functioning as determined by IQ and clinical response during treatment with atomoxetine.

Methods: The records of all the children and adolescents treated with atomoxetine at a university clinic in Catania, Italy, over a 3-year period were examined. A total of 55 clinically referred children and adolescents (aged 5-15 years, 53 males) with ADHD were treated with atomoxetine (10-110 mg/day; mean: $1.28 \mathrm{mg} / \mathrm{kg} /$ day) for a period ranging from 2 to 168 weeks (mean: $57.3 \pm \mathrm{SD}$ 39.4, median: 56). The IQ was assessed as part of the diagnostic evaluation prior to starting treatment. During treatment, clinical outcome was rated on the Clinical Global Impression-Improvement (CGI-I) and CGI-Severity (CGI-S) scales.

Results: The IQ ranged from 43 to 117 (mean: 80.6 \pm SD 18.6, median: 84). The IQ and final CGI-I scores were negatively correlated $(\mathrm{r}=-0.68$; $\mathrm{p}<0.01$ ). Children and adolescents with an IQ $<85$ were less likely to be responders (defined as a final CGI-I score of 1 or 2 ) than children and adolescents with an IQ $\geq 85$ (20.71\% vs $76.9 \%$; $p<0.001)$. None of the patients discontinued atomoxetine due to adverse effects, while treatment was discontinued in 20 subjects due to a lack of efficacy or ambivalence of parents about pharmacological treatment.

Conclusions: Atomoxetine appears to be less effective in children and adolescents with an IQ $<85$ than in children and adolescents in the average range of cognitive functioning. This difference is not accounted for by differences in the severity of ADHD symptoms, co-morbidity or reduced tolerability to the 
medication. These findings suggest that, in order to be fully informative, clinical trials of medications for ADHD should also include children and adolescents functioning in the borderline and cognitive disability range.

\section{Introduction}

The efficacy of atomoxetine in the treatment of attention-deficit hyperactivity disorder (ADHD) is supported by a number of controlled trials conducted in children with normal cognitive capacity. ${ }^{[1,2]}$ These studies indicate that atomoxetine, even though less effective than methylphenidate, decreases the symptoms of ADHD with a medium effect size compared with placebo. In clinical practice, atomoxetine is also commonly used to treat children and adolescents with an IQ in the range of intellectual disability $(\leq 70)$ or at the lower range of normality (between 71 and 84). Although there are indications that methylphenidate is less effective for children with developmental delays and associated cognitive impairment and that IQ moderates response to stimulant medication in children of normal intellect, ${ }^{[3]}$ it is unknown if IQ acts as a moderator of treatment effect also for atomoxetine.

A recently reported search for predictors of atomoxetine efficacy did not identify any demographics or clinical variables associated with treatment outcome. ${ }^{[4]}$ However, while these analyses included variables such as co-morbidity and type of ADHD, they did not account for the level of cognitive functioning as a possible predictor of response. In an open-label study in children with an IQ $<62$, atomoxetine was associated with significant improvement over baseline, with a symptomatic improvement in $88 \%$ of the patients, a positive response in $62 \%$ and remission or clinical normalization in $20 \%$. However, because of the lack of a control, possible treatment effects cannot be distinguished from time effects. ${ }^{[5]}$ A few studies of atomoxetine in children with pervasive developmental disorders, with response rates ranging from $60 \%$ to $75 \%$, have been reported, but two involved only participants with an IQ of at least $70,{ }^{[6,7]}$ and another, although placebo controlled, had a small sample size and did not report on possible effects of IQ on treatment response. ${ }^{[8]}$

We examined the records of all the children and adolescents treated with atomoxetine at the University of Catania Medical School Department of Child and Adolescent Neuropsychiatry, Catania, Italy, during a 3-year period. In Italy, stimulant medications are seldom used in the treatment of ADHD, which is primarily managed with behavioural interventions. If pharmacotherapy is warranted, parents often opt for atomoxetine. Based on the data on stimulants, we hypothesized that lower cognitive functioning as determined by a lower IQ would be associated with lower efficacy of the medication.

\section{Method}

\section{Sample Identification}

A systematic review of the medical records of all the children and adolescents treated with atomoxetine at the Child and Adolescent Department of Neuropsychiatry of the University of Catania, Catania, Italy, between January 2007 and July 2010 was conducted. The following information was extracted: demographics, IQ (routinely assessed in all patients as part of their intake evaluation), psychiatric diagnoses by the administration of the KiddieSchedule for Affective Disorders and SchizophreniaPresent and Lifetime Version (K-SADS-PL) ${ }^{[9]}$ interview to the parents, history of previous treatment, Parent Swanson, Nolan, and Pelham Rating Scale-IV (SNAP-IV) at baseline, clinician-rated Clinical Global Impression-Improvement (CGI-I) at endpoint and CGI-Severity (CGI-S) scales ${ }^{[10]}$ at baseline and through endpoint.

The ADHD diagnosis was made according to the DSM IV-TR criteria, while the K-SADS-PL was used to collect the clinical information. In addition, at least six of the inattention and/or hyperactivity/impulsivity items of the SNAP-IV 
had to be scored "pretty much" or higher for diagnosis accuracy.

The IQ was measured with the Wechsler Intelligence Scale for Children-III edition (WISC-III), ${ }^{[11]}$ and yielded, in addition to a full-scale total score (IQ), a verbal (VIQ) and a performance (PIQ) score. The WISC-III was used because the WISC-IV was not available in Italian.

\section{Assessments}

The SNAP-IV ${ }^{[12]}$ is a revision of the Swanson, Nolan and Pelham (SNAP) Questionnaire. The items from the DSM-IV criteria for ADHD are included in the SNAP-IV for the two subsets of symptoms: inattention (item numbers 1-9) and hyperactivity/impulsivity (item numbers 10-18). Also, items are included from the DSM-IV criteria for oppositional defiant disorder (ODD) [item numbers 19-26] since this disorder is often present in children and adolescents with ADHD. The SNAP-IV was used to characterize the severity of illness at baseline, but not for measuring treatment response.

Severity of illness and improvement during treatment were rated by the treating clinicians on the CGI-S and CGI-I scales. The CGI-S scale is a 7-point scale that requires the clinician to rate the current severity of the patient's illness ( $1=$ normal, not at all ill; $2=$ borderline mentally ill; $3=$ mildly ill; $4=$ moderately ill; $5=$ markedly ill; $6=$ severely ill; or $7=$ extremely ill). Likewise, the CGI-I scale requires the clinician to assess how much the patient's illness has improved or worsened relative to a baseline state on a 7-point scale $(1=$ very much improved; $2=$ much improved; $3=$ minimally improved; $4=$ no change; $5=$ minimally worse; $6=$ much worse; or $7=$ very much worse). Clinical response was defined as a final CGI-I score of 1 or 2. The raters were child neuropsychiatrists at the university clinic, who had been trained in the reliable use of these scales, as shown by scoring at least three tapes of clinical cases according to the gold standard.

\section{Data Analysis}

Standard descriptive statistics, chi-square or Fisher's exact tests, Pearson's r correlations and linear regression models were applied to the data in order to evaluate the association between the clinical and cognitive variables at baseline and during treatment. The association between cognitive level and clinical improvement was further explored after categorizing the sample based on an IQ below versus equal to or above one or two SDs below the normative mean (i.e. 85 and 70, respectively).

An $\alpha$ level of 0.05 was set for statistical significance. All the analyses were performed using the Statistical Package for Social Sciences (SPSS ${ }^{\circledR}$ 14.0 for Windows; distributed by IBM, Armonk, NY, USA).

\section{Results}

\section{Baseline Pre-Treatment Characteristics}

A total of 55 patients aged $5-15$ years ( 53 males, 2 females; IQ range: 43-117, mean: $80.6 \pm \mathrm{SD}$ 18.6, median: 84 ) with a DSM-IV ${ }^{[13]}$ diagnosis of ADHD (96\% with combined subtype and 4\% with hyperactive subtype) were treated with atomoxetine (table I). Twenty-six children and adolescents had an IQ $\geq 85$ (range: $85-117$, mean: $95.9 \pm 9.2$ ) and 29 children and adolescents had an IQ <85 (range: 43-84, mean: 66.9 \pm 13.3 ). Seventeen of the children and adolescents in the IQ $<85$ subgroup met criteria for intellectual disability; these children and adolescents had an IQ $\leq 70$ (range: $42-70$, mean: $57.9 \pm 9.7$ ), with a significant impairment clinically evaluated in adaptive behaviour in at least two domains. They did not differ from the rest of the sample with respect to age $(p=0.31)$, sex distribution $(p=0.53)$, SNAP-IV $(p=0.64)$ or CGI-S $(p=0.26)$ scores, or co-morbidity with ODD $(p=1.0)$. Moreover, 27 of all subjects (49\%) also met criteria for ODD, with no difference in the rate of co-morbid ODD between the IQ subgroups (13 patients with ODD in the subgroup with an IQ $\geq 85$ and 14 in those with an IQ <85).

No child met criteria for ADHD inattentive type, and the severity of inattentive symptoms, as measured on the SNAP-IV Inattentive Subscale, was similar across the IQ subgroups.

The patients had not received prior treatment with atomoxetine, stimulants or other psychotropic medications. All patients received behavioural 
Table I. Baseline demographics and clinical characteristics

\begin{tabular}{|c|c|c|c|c|}
\hline & All & $\mathrm{IQ}<85$ & $I Q \geq 85$ & $p-$ Value $^{a}$ \\
\hline$\overline{\mathrm{N}}$ & 55 & 29 & 26 & \\
\hline Sex (male: female) & $53: 2$ & $28: 1$ & $25: 1$ & 1.00 \\
\hline Age [y, mean (SD)] & $9.9(2.4)$ & $10.0(2.8)$ & $9.8(1.9)$ & 1.00 \\
\hline range & $5-15$ & $5-15$ & $6-13$ & \\
\hline IQ [mean (SD)] & $80.6(18.6)$ & $66.9(13.3)$ & $95.9(9.2)$ & \\
\hline range & $43-117$ & $43-84$ & $85-117$ & \\
\hline VIQ [mean (SD)] & $84.7(17.5)$ & $72.4(12.2)$ & $97.4(12.5)$ & \\
\hline range & $51-128$ & $51-91$ & $76-128$ & \\
\hline PIQ [mean (SD)] & $81.7(18.1)$ & $68.6(14.5)$ & $95.2(9.5)$ & \\
\hline range & $45-123$ & $45-106$ & $82-123$ & \\
\hline ADHD subtype $[\mathrm{n}(\%)]$ & & & & 1.00 \\
\hline inattentive & $0(0)$ & $0(0)$ & $0(0)$ & \\
\hline hyperactive/impulsive & $2(3.6)$ & $1(3.4)$ & $1(3.8)$ & \\
\hline combined & $53(96.36)$ & $28(96.55)$ & $25(96.15)$ & \\
\hline SNAP-IV [mean (SD)] & $41.1(5.8)$ & $41.2(6.0)$ & $41.0(5.7)$ & 0.91 \\
\hline range & $29-51$ & $30-51$ & $29-50$ & \\
\hline CGI-S [mean (SD)] & $5.5(0.9)$ & $5.6(0.9)$ & $5.38(0.8)$ & 0.31 \\
\hline range & $4-7$ & $4-7$ & $4-7$ & \\
\hline
\end{tabular}

a Student's t-test for continuous variables and Fisher's exact test for categorical variables.

$\mathbf{A D H D}=$ attention-deficit hyperactivity disorder; $\mathbf{C G I} \mathbf{S}=$ Clinical Global Impression-Severity Scale; $\mathbf{P I Q}=$ performance score; SNAP=Parent Swanson, Nolan, and Pelham Rating Scale; VIQ=verbal score.

interventions for the management of ADHD in addition to atomoxetine. None of these patients met criteria for an autism spectrum disorder, mood disorder or anxiety disorder according to DSM-IV-TR criteria or had a history of seizures.

No correlation between the IQ and SNAP-IV $(\mathrm{r}=-0.04 ; \mathrm{p}=0.77)$ or CGI-S $(\mathrm{r}=-0.14 ; \mathrm{p}=0.31)$ was detected. Age was not correlated with the IQ $(r=0.04 ; p=0.78)$, CGI-S $(r=-0.03 ; p=0.82)$, SNAP-IV Inattentive Subscale $(r=0.07 ; p=0.62)$ or SNAP-IV Hyperactivity/Impulsivity Subscale $(r=-0.25 ; p=0.07)$ scores. The subgroup with an IQ $<85(\mathrm{n}=29)$ was aged $5-15$ years (mean: $10.03 \pm$ 2.76 years), and the subgroup with an IQ $\geq 85$ $(n=26)$ was aged 6-13 years (mean: $9.77 \pm 1.94$ years); these subgroups did not differ significantly in age, gender or SNAP-IV scores (table I).

\section{Atomoxetine Treatment}

Atomoxetine was started at a dosage of $0.5 \mathrm{mg} / \mathrm{kg} /$ day and gradually increased based on clinical response. The mean duration of treatment was 57.3 weeks (SD 39.45, range: 2-168, median:
56 weeks), and did not differ between the IQ $\geq 85$ and IQ $<85$ subgroups. The mean final dosage was $1.28 \mathrm{mg} / \mathrm{kg} /$ day (SD 0.39, range: 0.32-1.76). The final dosage did not differ in the subgroup with an IQ $<85(1.29 \pm 0.49 \mathrm{mg} / \mathrm{kg} /$ day $)$ as compared with the subgroup with an IQ $\geq 85(1.27 \pm 0.31 \mathrm{mg} / \mathrm{kg} /$ day; $\mathrm{p}=0.86$ ). In the IQ $<70$ subgroup the duration of treatment $(43.76 \pm 27.98$ weeks; $p=0.07)$ and the final dosage $(1.21 \pm 0.59 \mathrm{mg} / \mathrm{kg} / \mathrm{day} ; \mathrm{p}=0.59)$ also did not differ from those in the IQ $>70$ subgroup (64.21 \pm 41.64 weeks; $1.30 \pm 0.30 \mathrm{mg} / \mathrm{kg} /$ day).

The most common adverse effects were as follows: loss of appetite $(n=13)$, abdominal pain $(n=12)$, nausea $(n=7)$, weight loss $(n=4)$, vomiting $(n=3)$, sleepiness $(n=2)$, paraesthesia $(n=1)$ and mydriasis $(n=1)$. These adverse effects were transient, and no patient discontinued treatment because of adverse effects. The rate or severity of adverse effects did not differ between the IQ subgroups.

The parents of 20 patients decided to discontinue treatment after relatively short periods of time, usually for lack of sufficient improvement and, in some cases, ambivalence about pursuing pharmacological treatment for their child. 


\section{IQ and Clinical Improvement}

The end of treatment CGI-I score was negatively correlated with the IQ $(\mathrm{n}=55, \mathrm{r}=-0.679 ; \mathrm{p}<0.01)$ [figure 1], as well as the VIQ $(\mathrm{r}=-0.61 ; \mathrm{p}<0.01)$ and the PIQ $(r=-0.69 ; \mathrm{p}<0.01)$. In the subgroup with an IQ $<85(n=29)$, the IQ was negatively correlated with final CGI-I $(r=-0.46 ; p=0.01)$ and CGI-S $(\mathrm{r}=-0.45 ; \mathrm{p}=0.02)$ scores. However, in the group with an IQ $\geq 85(n=26)$, there was no correlation between the IQ and CGI-I $(r=-0.036 ; p=0.86)$ or CGI-S $(r=0.105 ; p=0.61)$ scores.

Severity of illness scores showed a greater decline during treatment with atomoxetine in the IQ $\geq 85$ subgroup than in the IQ $<85$ subgroup (figure 2). Response, defined as a final CGI-I score of 1 or 2 , was more likely in children and adolescents with an IQ $\geq 85$ (20 of $26,76.9 \%$ ) than in those with an IQ $<85$ (6 of $29,20.7 \%$, Fisher's exact test: $\mathrm{p}<0.001)$. The response rate was even smaller in the subgroup functioning in the intellectual disability range (i.e. with an IQ $\leq 70$ ), with only 1 child of 17 being a responder, a rate that was significantly lower than that observed among children and adolescents with an IQ $>70$ ( 25 of $38,65.8 \%$, $\mathrm{X}^{2}=14.59 ; \mathrm{p}<0.01$; figure 3 ). Analyses of the response rates using the VIQ or the PIQ yielded similar results to those based on the full scale IQ.

\section{Discussion}

This is the first study, to our knowledge, that has examined the relationship between IQ and atomoxetine effectiveness. After systematically reviewing consecutively treated children and

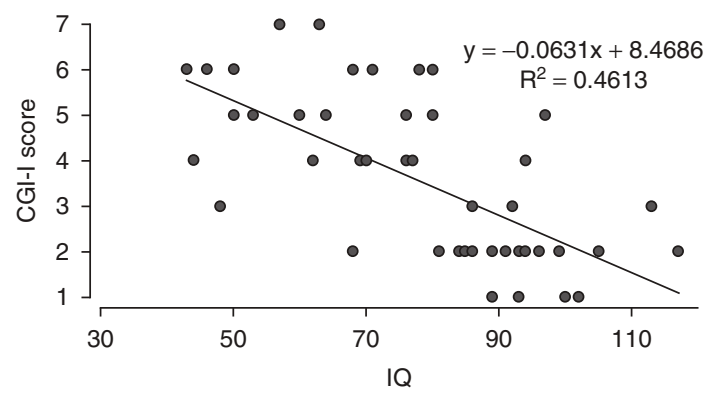

Fig. 1. $I Q$ and improvement during atomoxetine treatment. CGI-I = Clinical Global Impression-Improvement Scale.

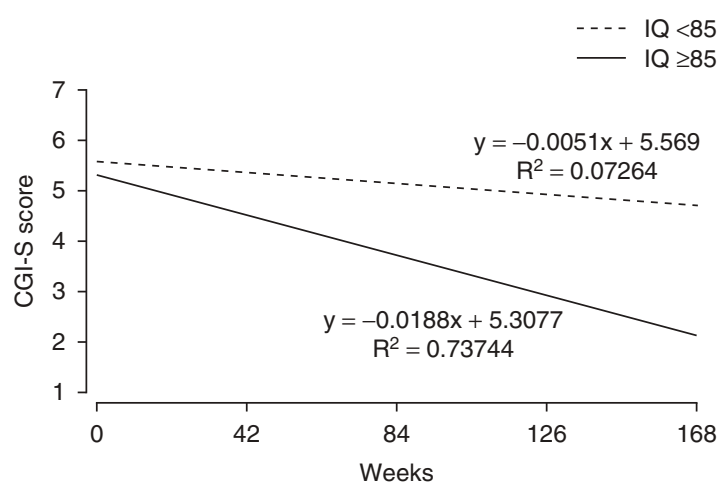

Fig. 2. Severity of illness estimated scores during atomoxetine treatment by $I Q$ subgroup. CGI-S=Clinical Global ImpressionSeverity Scale.

adolescents with ADHD, we found that atomoxetine was significantly less effective in patients with lower cognitive functioning (figures 1 and 2). Among children and adolescents with an IQ $\leq 70$, only $6 \%$ showed significant improvement (figure 3). The negative impact of IQ on treatment response was not evident in children and adolescents functioning in the average or above average range of intelligence. The effect of IQ on treatment response could not be explained by differences in the severity of ADHD, which was not related to the IQ at baseline.

It is possible that the methods used to ascertain ADHD symptoms may be less sensitive to detect treatment effects in children and adolescents with a lower IQ. Recognizing a clinical response in subjects with a lower IQ can be more challenging than in patients with a higher IQ. An improvement in attention and concentration may be masked by the functional impairment caused by the intellectual disability.

Our findings are consistent with reports that methylphenidate, a stimulant commonly used to treat ADHD, is less effective in children and adolescents with intellectual disabilities or pervasive developmental disorders. ${ }^{[14,15]}$ While one study reported that children with an IQ ranging from 50 to 74 responded to methylphenidate at a rate consistent with that observed in children with normal cognitive functioning, ${ }^{[16]}$ within the range of cognitive disability (i.e. an IQ $<70$ ), a lower IQ was found to predict poorer response to 


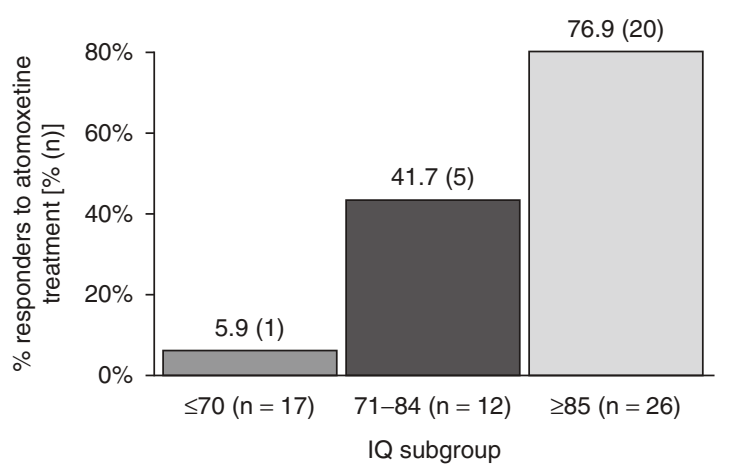

Fig. 3. Rate of response by IQ subgroup. Response: final Clinical Global Impression-Improvement Scale (CGI-I) score of 1 (very much improved) or 2 (much improved). This figure shows data from the full scale IQ; similar results were obtained using a verbal IQ or a performance IQ.

methylphenidate, as children with a moderate to severe degree of intellectual disability showed less improvement in ADHD symptoms than children with mild to borderline intellectual disability. ${ }^{[17]}$ The IQ was also found to be a moderator of response to treatment with methylphenidate in children with ADHD and an IQ of $\geq 80$, in the direction of a better response with a higher IQ. ${ }^{[3]}$ These data suggest that pharmacological treatment of ADHD is in general less effective in children with a lower IQ, and that this finding is not specific to atomoxetine. In our sample, however, the IQ did not influence the tolerability to atomoxetine, while methylphenidate has been reported to be more likely to induce intolerable adverse effects in children with autism and a low IQ $(18 \%)$ than in children with ADHD who are otherwise normally developing. ${ }^{[15]}$

There may be specific biological differences underlying differences in efficacy by IQ. Atomoxetine acts through selectively increasing dopamine transmission at the prefrontal cortex level, ${ }^{[18]}$ and several studies have shown a dysfunction in the dopaminergic and adrenergic transmission in the prefrontal cortex of individuals with idiopathic intellectual disabilities. ${ }^{[19]}$ It is possible that these abnormalities impair the efficacy of pharmacological treatment.

The present study has a number of limitations that must be taken into account in interpreting the data. First, this was a retrospective analysis of naturalistically treated children and adolescents. Even though these patients were consecutively treated at a specialized neuropsychiatric clinic, the uncontrolled nature of the study prevents conducting moderator analyses. Second, the sample size was relatively small. Third, the patients were derived from a homogeneous ethnic background and geographical context, all coming from a single university clinic, and may not represent other clinical settings. Finally, although parents provided detailed information on school performance and behaviour, teacher SNAP-IV scores were not consistently obtained to assess drug response accurately. Despite these important limitations, a clear-cut inverse relationship between IQ and degree of improvement was evident.

These findings may have important implications for both clinical practice and further research. Atomoxetine is often used for the treatment of children and adolescents with ADHD in the context of a low IQ. Clinicians should be alert to the possibility that the medication may be less likely to be effective in these patients. As this situation does not seem to be specific to atomoxetine, but also to stimulant medications, there may be structural differences between ADHD in individuals with low cognitive functioning versus those with ADHD and a higher IQ. While psychometrically valid rating scales, such as the Aberrant Behavior Checklist, ${ }^{[20]}$ are available for children with intellectual impairment, and novel approaches focused on individually targeted symptoms have been introduced, ${ }^{[21]}$ research to develop more sensitive and specific assessment instruments for evaluating ADHD symptoms in children and adolescents with a lower IQ may be warranted. Regulatory clinical trials for ADHD medications typically exclude children with a lower IQ. However, extrapolating data from these trials to children with a lower IQ may overestimate the effectiveness of medications in these patients.

\section{Conclusions}

A lower IQ among children and adolescents with ADHD was found to be strongly associated with decreased benefit from atomoxetine treatment. If replicated, these findings suggest that, in 
order to be fully informative for clinical practice, future trials of medications for ADHD should study subjects with a broad range of cognitive abilities, including also children and adolescents with a lower IQ and cognitive disabilities.

\section{Acknowledgements}

Luigi Mazzone and Laura Reale contributed equally to this work in terms of realization of the study, data analysis and paper writing. Valeria Mannino and Mariadonatella Cocuzza collected the data, and Benedetto Vitiello contributed to the design of the study, supervised statistical analysis and helped in the theoretical interpretation of the results. This study was funded by the Division of Child Neurology and Psychiatry of the University of Catania, Catania, Italy. Dr Mannino received educational travel funds by Eli Lilly. The other authors (LM, LR, BV) have no relevant financial disclosures.

\section{References}

1. Michelson D, Faries D, Wernicke J, et al., Atomoxetine ADHD Study Group. Atomoxetine in the treatment of children and adolescents with attention-deficit/hyperactivity disorder: a randomized, placebo-controlled, dose-response study. Pediatrics 2001; 108 (5): E83

2. Newcorn JH, Kratochvil CJ, Allen AJ, et al., Atomoxetine/ Methylphenidate Comparative Study Group. Atomoxetine and osmotically released methylphenidate for the treatment of attention deficit hyperactivity disorder: acute comparison and differential response. Am J Psychiatry 2008 Jun; 165 (6): 721-30

3. Owens EB, Hinshaw SP, Kraemer HC, et al. Which treatment for whom for ADHD? Moderators of treatment response in the MTA. J Consult Clin Psychology 2003; 71 (3): 540-52

4. Scott NG, Ripperger-Suhler J, Rajab MH, et al. Factors associated with atomoxetine efficacy for treatment of attentiondeficit/hyperactivity disorder in children and adolescents. J Child Adolesc Psychopharmacol 2010; 20 (3): 197-203

5. Fernandez-Jaen A, Fernandez-Mayoralas DM, Perez BC, et al. Atomoxetine for attention deficit hyperactivity disorder in mental retardation. Pediatr Neurol 2010; 43 (5): 341-7

6. Posey DJ, Wiegand RE, Wilkerson J, et al. Open-label atomoxetine for attention deficit/hyperactivity disorder symptoms associated with high-functioning pervasive developmental disorders. J Child Adolesc Psychopharmacol 2006; 16 (5): 599-610

7. Troost PW, Steenhuis MP, Tuyman-Qua HG, et al. Atomoxetine for attention-deficit/hyperactivity disorder symptoms in children with pervasive developmental disorders: a pilot study. J Child Adolesc Psychopharmacol 2006; 16 (5): 611-9

8. Arnold LE, Aman MG, Cook AM, et al. Atomoxetine for hyperactivity in autism spectrum disorders: placebocontrolled crossover pilot trial. J Am Acad Child Adolesc Psychiatry 2006; 45 (10): 1196-205
9. Kaufman J, Birmaher B, Brent D, et al. Schedule for Affective Disorders and Schizophrenia for School-Age Children-Present and Lifetime Version (K-SADS-PL): initial reliability and validity data. J Am Acad Child Adolesc Psychiatry 1997; 36: 980-8

10. Guy W, editor. ECDEU assessment manual for psychopharmacology: publication ADM 76-338. Washington, DC: US Department of Health, Education and Welfare, 1996: 218-22

11. Wechsler D. Wechsler intelligence scale for children, WISC-III. 3rd ed. New York (NY): Psychological Corporation, 1991

12. Swanson JM, Sandman CA, Deutsch C, et al. Methylphenidate hydrochloride given with or before breakfast: I. Behavioral, cognitive, and electrophysiologic effects. Pediatrics 1983; 72: 49-55

13. American Psychiatric Association. Diagnostic and statistical manual of mental disorders. 4th ed., text revision. Washington, DC: American Psychiatric Association, 2002

14. Aman MG, Buican B, Arnold LE. Methylphenidate treatment in children with borderline IQ and mental retardation: analysis of the three aggregated studies. J Child Adolesc Psychopharmacol 2003; 13: 28-40

15. Research Units on Pediatric Psychopharmacology (RUPP) Autism Network. Randomized, controlled, crossover trial of methylphenidate in pervasive developmental disorders with hyperactivity. Arch Gen Psychiatry 2005; 62: 1266-74

16. Handen BL, Breaux AM, Gosling A, et al. Efficacy of methylphenidate among mentally retarded children with attention deficit hyperactivity disorder. Pediatrics 1990; 86 (6): 922-30

17. Aman MG, Marks RE, Turbott SH, et al. Methylphenidate and thioridazine in the treatment of intellectually subaverage children: effects on cognitive-motor performance. J Am Acad Child Adolesc Psychiatry 1991; 30 (5): 816-24

18. Bymaster FP, Katner JS, Nelson DL, et al. Atomoxetine increases extracellular levels of norepinephrine and dopamine in prefrontal cortex of rat: a potential mechanism for efficacy in attention deficit/hyperactivity disorder. Neuropsychopharmacology 2002 Nov; 27 (5): 699-711

19. Diamond A. Consequences of variations in genes that affect dopamine in prefrontal cortex. Cereb Cortex 2007 Sep; 17 Suppl. 1: i161-70

20. Marshburn EC, Aman MG. Factor validity and norms for the aberrant behavior checklist in a community sample of children with mental retardation. J Autism Dev Disord 1992; 22: 357-73

21. Arnold LE, Vitiello B, McDougle C, et al. Parent-defined target symptoms respond to risperidone in RUPP autism study: customer approach to clinical trials. J Am Acad Child Adolesc Psychiatry 2003; 42: 1443-50

Correspondence: Dr Luigi Mazzone, Division of Child Neurology and Psychiatry, Department of Pediatrics, University of Catania, Via S. Sofia No. 78, Catania, 95100, Italy.

E-mail: gigimazzone@yahoo.it 